\title{
NOT ALL OF THE RARE OPERATIONAL TAXONOMIC UNITS (OTUS) PLAY THE SAME ROLE IN MAINTAINING COMMUNITY STABILITY
}

\author{
WU, L. ${ }^{1 * \#}-\mathrm{SUN}, \mathrm{Q}^{2 \#}-\mathrm{NI}, \mathrm{J}^{3,4 *}$ \\ ${ }^{1}$ School of Life Science, Hefei Normal University, Hefei 230601, China \\ (phone: +86-551-6367-4150; fax: +86-551-6367-4150) \\ ${ }^{2}$ School of Resources and Environment Engineering, Anhui University, Hefei 230039, China \\ (phone: +86-551-6386-1882; fax: +86-551-6367-4150) \\ ${ }^{3}$ Guangdong Provincial Key Laboratory of Microbial Culture Collection and Application, \\ Guangdong Institute of Microbiology, Guangzhou 510070, China \\ (phone: +86-020-8768-4471; fax: +86-020-8768-4471)
}

${ }^{4}$ State Key Laboratory of Applied Microbiology Southern China, Guangzhou 5100770, China (phone: +86-020-8768-4471; fax: +86-020-8768-4471)

*Corresponding authors

e-mail:wuli090121@126.com (Wu, L.)

e-mail:nijiajia2005@126.com (Ni, J.)

${ }^{\#} W U$, L. and SUN, Q. have contributed equally to this work.

(Received 15 ${ }^{\text {th }}$ Jul 2016; accepted $12^{\text {th }}$ Oct 2016)

\begin{abstract}
The role of rare biosphere in maintaining the community diversity and metabolic activity has recently been highlighted. However, it is still unclear whether the rare species play the same role in maintaining the community diversity. Considering different responses of microbial species on environment changes, we speculate rare species played different roles in maintaining the $\alpha$-diversity. To verify the speculation, we analyzed the prokaryotic species in three eutrophic rivers and one eutrophic lake via Miseq sequencing of $16 \mathrm{~S}$ rDNA amplicons. Although more than 50 phyla were identified from 20 samples, only seven were dominant. The dominant species were strictly restricted. The rare operational taxonomic units (OTUs) belonging to the dominant phyla played a crucial role in maintaining the community diversity. These results intensified our knowledge on the role of rare species in maintaining the diversity of microbial community.
\end{abstract}

Keywords: Chaohu Lake; community diversity; microbial ecology; rare species; stochastic

\section{Introduction}

Enumerating microbial community diversity (MCD) and analyzing the mechanisms that maintain the diversity are two basic ecological and environmental issues. The application of DNA-based molecular tools, especially high-throughput sequencing technologies, greatly enhances our understanding of the MCD as these techniques circumvent the problems in classical culture-based techniques (Caporaso et al., 2011; Rees et al., 2004; Sergeant et al., 2012). Lots of microbial communities that exist in various habitats, such as soil (Sul et al., 2011; Vasileiadis et al., 2012), ocean (Gilbert et al., 2009), human and animal guts (David et al., 2014; Lee et al., 2011; Ni et al., 2014) and freshwater (Kara et al., 2013; Widder et al., 2014), have been investigated by these 
techniques. Based on the investigations, a lot of ecological principles that maintain the MCD are described, such as the taxa-area relationship (Horner-Devine et al., 2004), the cosmopolitan distribution of microbial subgroups (Chaffron et al., 2010) and the distance-decay relationship (Bell, 2010). These ecological principles extend our knowledge about the mechanisms that maintain the MCD (Ni et al., 2016). However, although we know microbial communities are extremely diverse in natural environments, and they are typically composed of a few dominant species followed by a large number of rare species (Zhou et al., 2015), the roles of the rare species on maintaining the community $\alpha$-diversity are still largely unclear.

Recently, the role of rare species on maintaining the MCD and the metabolic activity has been highlighted (Campbell et al., 2011; Coveley et al., 2015). Coveley et al. (2015) point out a fraction of the rare species acts as a backup system for maintaining ecosystem resilience in face of perturbation. When environment is disturbed, some rare species rise to dominant species to maintain the diversity and metabolic activity of microbial community. However, whether every rare species in a specific microbial community plays the same role on maintaining the MCD is still unclear.

Shannon's index and Simpson index are the generally accepted indexes to indicate the community $\alpha$-diversity. Since both Shannon's index and Simpson index consider the abundance of each species, the influences of species with different relative abundances are distinct. Therefore, when the dominant species is absent causing by environmental disturbance, the ability becoming to dominant species was a rational agent to character the role of rare species on maintaining the community $\alpha$-diversity. According to this inference, the rare species that can become to dominant species under environmental disturbance play more important roles than those cannot become to dominant species. Therefore, through analyzing the fluctuation of species in different habitats, we can infer the roles of rare species on maintaining the $\alpha$-diversity.

To address the question that whether every rare species in a specific microbial community plays the same role on maintaining the $\alpha$-diversity, we analyzed the fluctuation of prokaryotic species in three eutrophic rivers and one eutrophic lake via Miseq sequencing of prokaryotic 16S rRNA gene amplicons.

\section{Materials and Methods}

\section{Sampling sites and sample collection}

Samples were collected in four seasons: September 2013 (autumn), December 2013 (winter), March 2014 (spring), and June 2014 (summer). In each season, five sites were set at Nanfei River (NF, N31 ${ }^{\circ} 51^{\prime} 00.11^{\prime \prime}$, E117 $\left.18^{\prime} 34.27^{\prime \prime}\right)$, Zhigao River (ZG, N31 466'32.77", E11744'55.04"), Hangbu River (HB, N31²5'19.08", E11656'48.32"), East Chaohu Lake (EC, E117 35'52.2", N31 36'21.0") and West Chaohu Lake (WC, E117 17'44.7", N31 40'26.5") (Fig. 1). All sites were sampled within one day. Water of $500 \mathrm{ml}$ and water of $1 \mathrm{~L}$ was collected from each sampling site for chemical characterization and for microbial DNA extraction, respectively. DNA extraction was carried out within $24 \mathrm{~h}$ after sampling collection. 


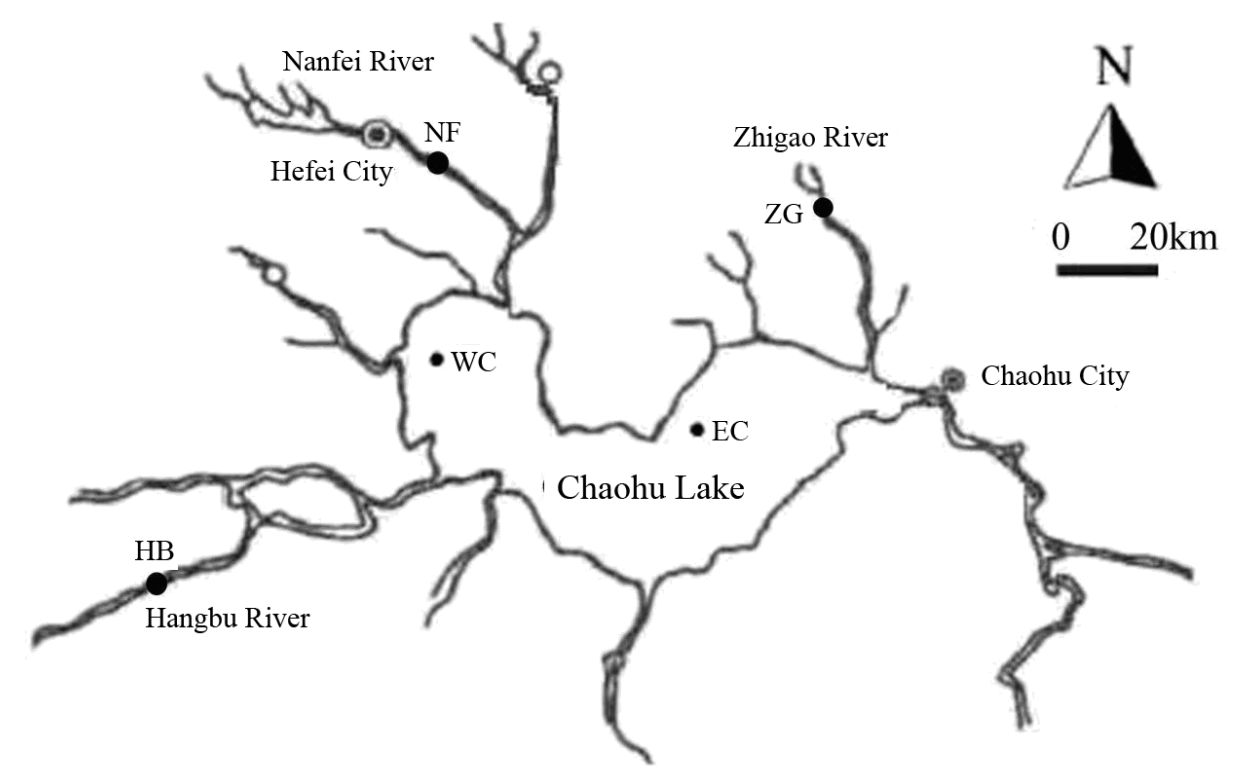

Figure 1. Sampling sites of Chaohu Lake and three rivers. ZG, Zhigao River; HB, Hangbu River; NF, Nanfei River; WC, West Chaohu Lake and EC, East Chaohu Lake.

\section{Measurement of physical and chemical indexes}

Water temperature (WT) was measured in at field simultaneously with sample collection. Total phosphorus (TP), $\mathrm{pH}$, total nitrogen $(\mathrm{TN})$, nitrate $\left(\mathrm{NO}_{3}-\mathrm{N}\right)$ and ammonia nitrogen $\left(\mathrm{NH}_{4}-\mathrm{N}\right)$ were measured according to standard methods (Huang, 2000). The trophic level index (TLI) was calculated based upon the concentration of TP according to the standard method (OECD, 1982).

\section{DNA extraction and MiSeq sequencing of 16S rRNA gene amplicons}

The microbial DNA was extracted using the standard phenol-chloroform method with some modifications (Ni et al., 2010). The water samples for microbial DNA extraction were filtered by polycarbonate membranes with $0.22 \mu \mathrm{m}$ pore size. The filter membranes were cut into pieces and transferred into $2.0 \mathrm{ml}$ sterile centrifuge tubes. Then the tubes were added $600 \mu \mathrm{L}$ of extraction buffer $(100 \mathrm{mM}$ Tris- $\mathrm{HCl}(\mathrm{pH} 8.0), 100$ $\mathrm{mM}$ phosphate (pH 8.0), $100 \mathrm{mM}$ EDTA (pH 8.0), $150 \mathrm{mM} \mathrm{NaCl}, 1 \%$ (wt/vol) CTAB and $2 \mathrm{mg}$ Lysozyme) and were incubated at $37^{\circ} \mathrm{C}$ for $90 \mathrm{~min}$. After incubation, cells were disrupted by three cycles of freezing $\left(-80^{\circ} \mathrm{C}\right.$ for $\left.30 \mathrm{~min}\right)$ and thawing $\left(65^{\circ} \mathrm{C}\right.$ for 30 min). Cells were then incubated at $65^{\circ} \mathrm{C}$ for 30 min with $100 \mu \mathrm{L} 20 \%$ SDS and $10 \mathrm{mg}$ proteinase $\mathrm{K}$. The supernatant was treated with 0.2 volumes $8 \mathrm{M}$ potassium acetate to remove polysaccharides followed by treatment with an equal volume of phenolchloroform-isoamyl alcohol $(25: 24: 1)$ to remove protein and cell debris. Residual phenol was removed by the addition of an equal volume of chloroform-isoamyl alcohol. Nucleic acids were precipitated from the supernatant by adding approximately an equal volume of isopropanol and maintaining the samples at $4^{\circ} \mathrm{C}$ for at least $2 \mathrm{~h}$. The solution was centrifuged at $14,000 \times \mathrm{g}$ for $20 \mathrm{~min}$. Residual of isopropanol were completely removed by adding $70 \%(\mathrm{v} / \mathrm{v})$ alcohol and centrifugation at $14,000 \times \mathrm{g}$ for $20 \mathrm{~min}$. Extracted genomic DNA samples were dissolved by $50 \mu \mathrm{L}$ of Tris-EDTA (TE) buffer. DNA concentration and quality were checked using a NanoDrop Spectrophotometer. 
The extracted DNA was diluted to $10 \mathrm{ng} / \mu \mathrm{L}$ and stored at $-40^{\circ} \mathrm{C}$ for downstream use.

V4-V5 hypervariable region of prokaryotic 16S rDNA was amplified using universal primer 515F (5'-GTGCCAGCMGCCGCGGTAA-3') and 909R (5'CCCCGYCAATTCMTTTRAGT -3') with $12 \mathrm{nt}$ unique barcode and sequenced as a previous report ( $\mathrm{Li}$ et al., 2016). The sequencing was performed on an Illumina Miseq system using paired-end technology provided by Dongguan Meikang BioScience Inc., China.

\section{Data analysis}

The raw reads were spliced using FLASH 1.2.8. The merged sequences were processed using QIIME Pipeline (Caporaso et al., 2010). All sequence reads were trimmed and assigned to each sample based on their barcodes. The sequences with high quality (length $>300 \mathrm{bp}$, without ambiguous base ' $\mathrm{N}$ ', and average base quality score $>$ 30) were used for downstream analysis. Chimera check was conducted using the Uchime algorithm (Edgar et al., 2011). Non-chimera sequences were clustered into operational taxonomic units (OTUs) at a 97\% identity threshold. Taxonomy was assigned using the Ribosomal Database Project classifier (Wang et al., 2007). Canonical correspondence analysis (CCA) with Monte Carlo permutation test was carried out by vegan package in $\mathrm{R}$ platform (Dixon, 2003).

The merged sequences were deposited into the NCBI short-reads archive database (Accession Number: SRR2079561-SRR2079595 and SRR2079602).

\section{Results and Discussion}

A total of 320554 high-quality sequences from 20 samples were obtained in this study. At the phylum level, except $0.7-3.8 \%$ reads were unclassified into archaeal and bacterial phyla, 2 distinct archaeal phyla and 49 distinct bacterial phyla were identified. However, only seven bacterial phyla, Actinobacteria, Bacteroidetes, Cyanobacteria, Firmicutes, Planctomycetes, Proteobacteria and Verrucomicrobia were dominant phyla (their relative abundance is more than 10\%), and their reads occupied $88.7-95.7 \%$ of total reads. Four of these seven phyla, i.e. Actinobacteria (13 sampling sites), Bacteroidetes (14 sampling sites), Cyanobacteria (14 sampling sites) and Proteobacteria (20 sampling sites) were the dominant phyla in more than ten sampling sites. These results implied that although there were high biodiversity, the dominant phyla in eutrophic rivers and lake were strictly restricted. In addition, there was no consistent seasonal changing pattern among different sampling sites. For instance, in the sampling site ZG, the relative abundance of Verrucomicrobia in winter was obviously higher than other seasons, while those in autumn were obviously higher than others in the sampling site HB (Fig. 2).

To analyze the randomness and succession of microorganisms in species (OTU) level, we compared the trends of the dominant OTUs (their relative abundance is more than $1 \%$ ) among different seasons. A total of 14,746 OTUs were detected from the 20 sampling sites. However, only 83 OTUs were dominant. All of the dominant OTUs were classified into Bacteria. Except one dominant OTU was classified into phylum Chlorobi and one dominant OTU was not classified into any phylum, others were classified into the seven dominant phyla, i.e. Actinobacteria (7 OTUs), Bacteroidetes (18 OTUs), Cyanobacteria (15 OTUs), Firmicutes (2 OTUs), Planctomycetes (4 OTUs), 
Proteobacteria (29 OTUs) and Verrucomicrobia (6 OTUs) (Fig. 3). It was consistent with the dominant phyla. The result showed that even though there were more than 14,700 OTUs detected in the present study, the dominant OTUs were strictly restricted into the seven dominant phyla. This result implied that although the role of rare species in maintaining the community diversity has been highlighted in a highly diverse ecosystem (Coveley et al., 2015), it did not mean that all of the rare OTUs played the same role in maintaining the community $\alpha$-diversity. The rare species belong to the seven dominant phyla were more important in maintaining the community $\alpha$-diversity. The role of those rare OTUs that were not classified into the seven dominant phyla still needs to illustrate.

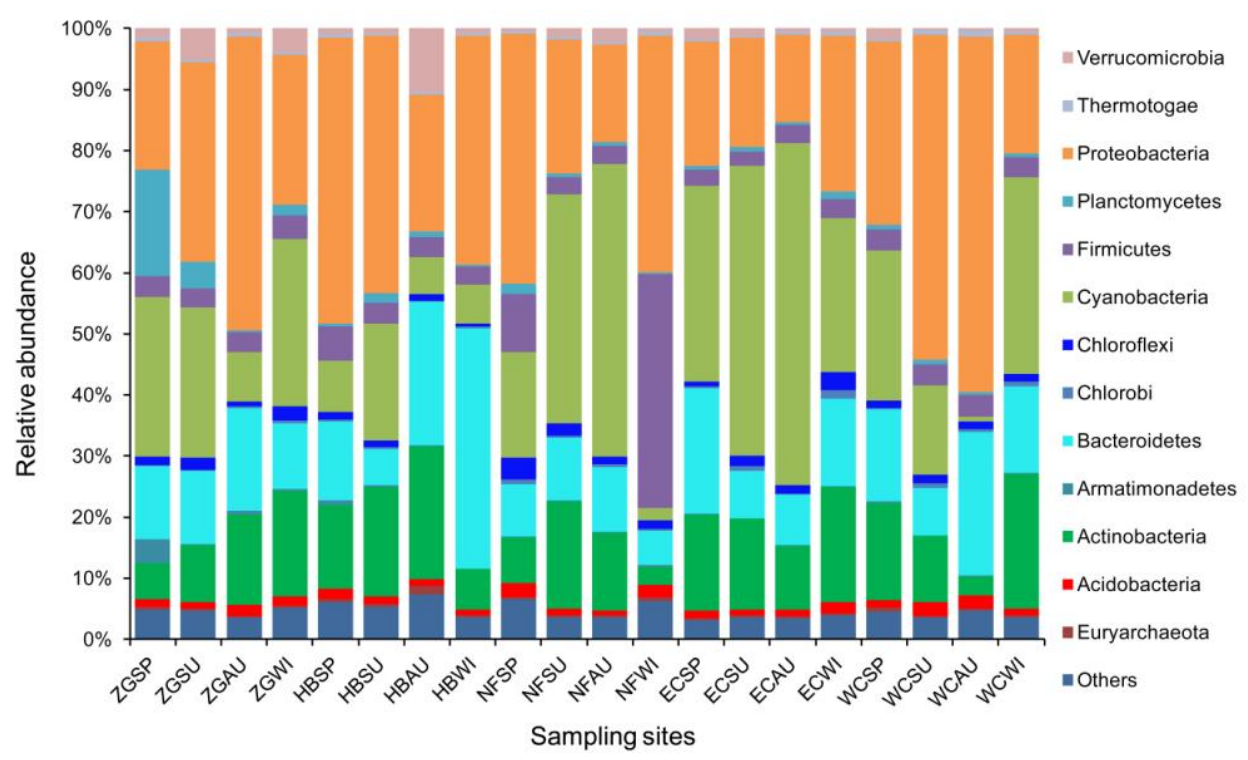

Figure 2. Relative abundances of prokaryotic phyla harboring in eutrophic rivers and Lake Chaohu. ZG, Zhigao River; HB, Hangbu River; NF, Nanfei River; WC, West Chaohu Lake and EC, East Chaohu Lake.

To elucidate the reasons causing the turnover of dominant OTUs, we analyzed the relevance between the compositions of the dominant OTUs and the environmental physicochemical factors containing WT, pH, TN, NH4, NO3, TP and TLI. According to the TLI, ZGWI, HBWI, WCWI, WCSP were eutrophic, the other sites were hypereutrophic. CCA with Monte Carlo permutation test showed neither total of environmental factors (Permutation test with 999 permutations, Pseudo- $F=1.058, p=$ 0.404 ) nor each environmental factor significantly impacted on the dominant OTU turnover.

To analyze fluctuating details of the dominant OTUs, we artificially differentiated the dominant OTUs between two distinct factions according to their occurrence frequency as dominant OTU in the samples: high-frequency dominant OTUs that appear more than or equal half number of samples $(\geq 10)$ with the relative abundance more than $1 \%$ and low-frequency dominant OTUs that appear less than half number of samples $(<10)$ with the relative abundance more than $1 \%$. A total of 5 OTUs were detected as high-frequency dominant OTUs (Fig. 4) and they belonged to different phyla, i.e. each two OTUs belonged to Proteobacteria and Actinobacteria, and one OTU belonged to 
Cyanobacteria. Seventy-eight OTUs were detected as low-frequency dominant OTUs, in which 43 dominant OTUs were only detected from one samples (Fig. 4). This result implied that stochastic process was a non-ignorable factor that influences the microbial community structure, just as other evidences provided by previous reports (Chase, Myers, 2011; Zhou et al., 2014).

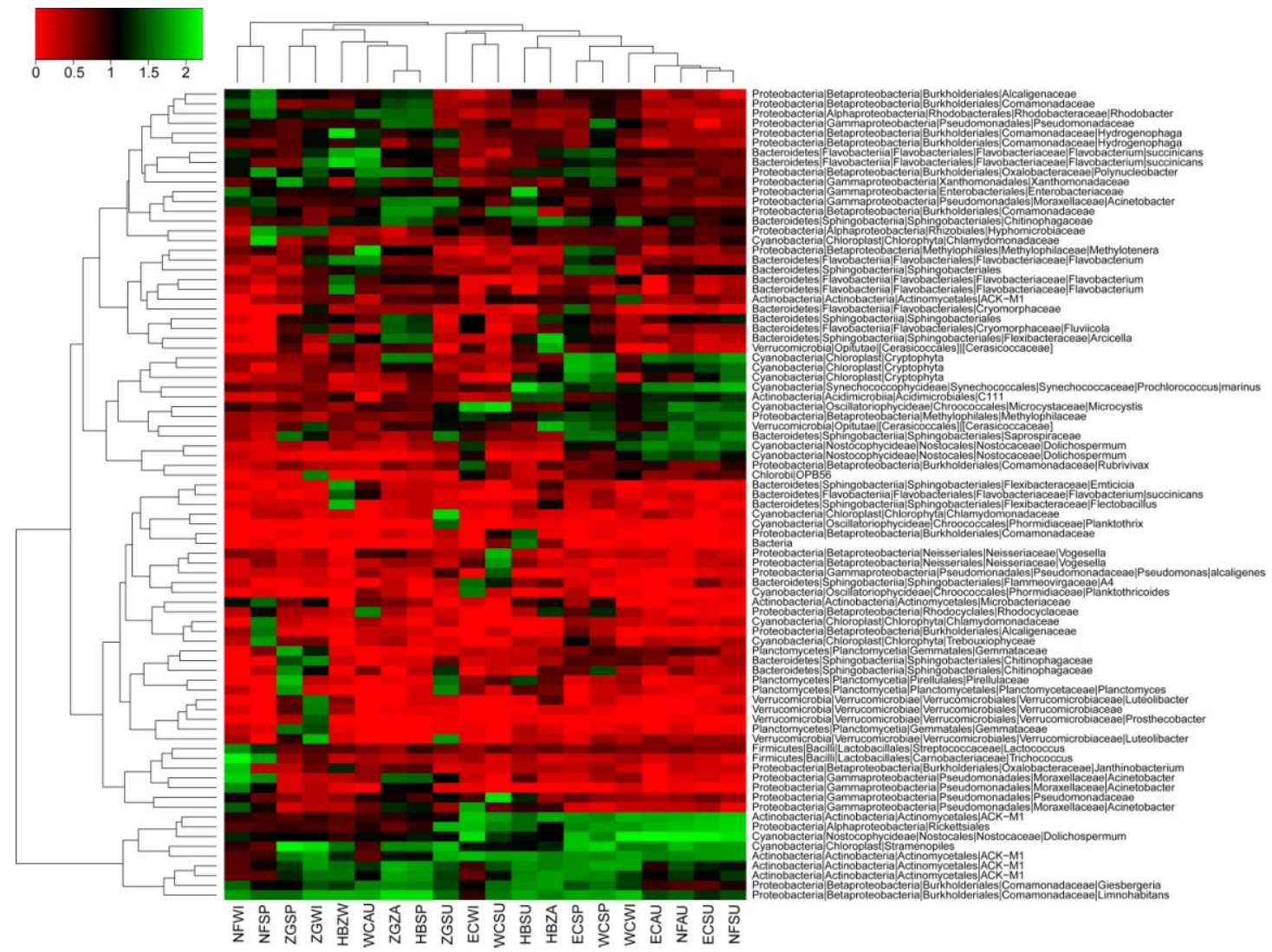

Figure 3. Heatmap profile of dominant OTUs. The data were transformed according to the formula as follows, $\log _{10}$ (relative abundance of each otu $\left.* 100+1\right)$. ZG, Zhigao River; $H B$, Hangbu River; NF, Nanfei River; WC, West Chaohu Lake and EC, East Chaohu Lake.

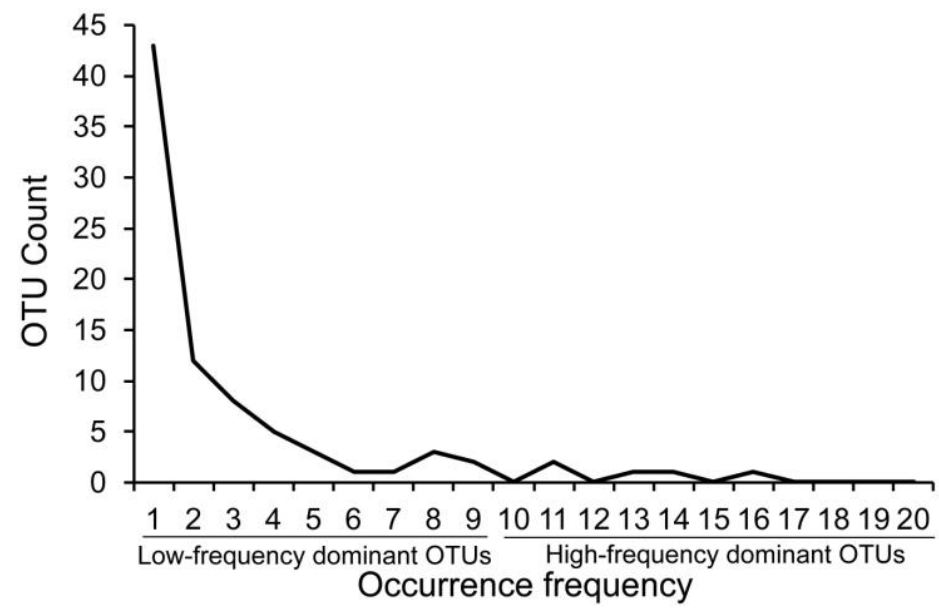

Figure 4. Occurrence frequency of the dominant OTUs in the samples 


\section{Conclusions}

It was concluded that not all of the rare OTUs played the same role in maintaining the community diversity. The rare OTUs belonging to seven dominant phyla played a crucial role in maintaining the microbial community diversity and which OTUs in the seven dominant phyla could become the dominant OTUs were conditionally stochastic.

Acknowledgements. This research was supported by the Natural Science Foundation of Anhui Province (No.1208085QC61), the Foundation for Young Talents in College of Anhui Province (No. 2012SQRL164ZD), the Nature Science Foundation of China (No. 31500417), the Guangdong Natural Science Foundation (No. 2014A030310281) and the Youth Scientific Research Foundation of Guangdong Academy of Sciences (No. qnjj201504).

\section{REFERENCES}

[1] Bell, T. (2010): Experimental tests of the bacterial distance-decay relationship. - ISME Journal 4: 1357-1365.

[2] Campbell, B. J., Yu, L., Heidelberg, J. F., Kirchman, D. L. (2011): Activity of abundant and rare bacteria in a coastal ocean. - Proceeding of the National Academy of Sciences of the United States of America 108: 12776-12781.

[3] Caporaso, J. G., Kuczynski, J., Stombaugh, J., Bittinger, K., Bushman, F. D., Costello, E. K., Fierer, N., Peña, A. G., Goodrich, J. K., Gordon, J. I., Huttley, G. A., Kelley, S. T., Knights, D., Koenig, J. E., Ley, R. E., Lozupone, C. A., McDonald, D., Muegge, B. D., Pirrung, M., Reeder, J., Sevinsky, J. R., Turnbaugh, P. J., Walters, W. A., Widmann, J., Yatsunenko, T., Zaneveld, J., Knight, R. (2010): QIIME allows analysis of high-throughput community sequencing data. - Nature Methods 7: 335-336.

[4] Caporaso, J. G., Lauber, C. L., Walters, W. A., Berg-Lyons, D., Lozupone, C. A., Turnbaugh, P. J., Fierer, N., Knight, R. (2011): Global patterns of 16S rRNA diversity at a depth of millions of sequences per sample. - Proceeding of the National Academy of Sciences of the United States of America 108: 4516-4522.

[5] Chaffron, S., Rehrauer, H., Pernthaler, J., von Mering, C. (2010): A global network of coexisting microbes from environmental and whole-genome sequence data. - Genome Research 20: 947-59.

[6] Chase, J. M., Myers, J. A. (2011): Disentangling the importance of ecological niches from stochastic processes across scales. - Philosophical Transactions of the Royal Society B Biological Sciences 366: 2351-2363.

[7] Coveley, S., Elshahed, M. S., Youssef, N. H. (2015): Response of the rare biosphere to environmental disturbance in a highly diverse ecosystem (Zodletone spring, OK, USA). PeerJ 3: e1182.

[8] David, L. A., Maurice, C. F., Carmody, R. N., Gooenberg, D. B., Button, J. E., Wolfe, B. E., Ling, A. V., Devlin, A. S., Varma, Y., Fischbach, M. A., Biddinger, S. B., Dutton, R. J., Turnbaugh, P. J. (2014): Diet rapidly and reproducibly alters the human gut microbiome. Nature 505: 559-563.

[9] Dixon, P. (2003): VEGAN, a package of R functions for community ecology. - Journal of Vegetation Science 14: 927-930

[10] Edgar, R. C., Haas, B. J., Clemente, J. C., Quince, C., Knight, R. (2011): UCHIME improves sensitivity and speed of chimera detection. - Bioinformatics 27: 2194-2200.

[11] Gilbert, J. A., Field, D., Swift, P., Newbold, L., Oliver, A., Smyth, T., Somerfield, P. J., Huse, S., Joint, I. (2009): The seasonal structure of microbial communities in the Western English Channel. - Environmental Microbiology 11: 3132-3139.

[12] Horner-Devine, M. C., Lage, M., Hughes, J. B., Bohannan, B. J. M. (2004): A taxa-area 
relationship for bacteria. - Nature 432: 750-753.

[13] Huang, X. F. (2000): Survey, Observation and Analysis of Lake Ecology. - Standard Press of China, Beijing

[14] Kara, E. L., Hanson, P. C., Hu, Y. H., Winslow, L., McMahon, K. D. (2013): A decade of seasonal dynamics and co-occurrences within freshwater bacterioplankton communities from eutrophic Lake Mendota, WI, USA. - ISME Journal 7: 680-684.

[15] Lee, J. E., Lee, S., Sung, J., Ko, G. (2011): Analysis of human and animal fecal microbiota for microbial source tracking. - ISME Journal 5: 362-365.

[16] Li, H., Qu, J., Li, T., Li, J., Lin, Q., Li, X. (2016): Pika population density is associated with the composition and diversity of gut microbiota. - Frontiers in Microbiology 7: 758.

[17] Ni, J. J., Xu, M., He, Z., Sun, G., Guo, J. (2016): Novel insight into evolutionary process from average genome size in marine bacterioplanktonic biota. - Applied Ecology and Environmental Research 14: 65-75.

[18] Ni, J. J., Yan, Q. Y., Yu, Y. H., Zhang, T. L. (2014): Factors influencing the grass carp gut microbiome and its effect on metabolism. - FEMS Microbiology Ecology 87: 704-714.

[19] Ni, J. J., Yu, Y. H., Feng, W. S., Yan, Q. Y., Pan, G., Yang, B., Zhang, X., Li, X. M. (2010): Impacts of algal blooms removal by chitosan-modified soils on zooplankton community in Taihu Lake, China. - Journal of Environmental Sciences 22: 1500-1507.

[20] OECD (1982): Eutrophication of Waters: Monitoring, Assessment and Control. - OECD publications and Information Center, Paris

[21] Rees, G. N., Baldwin, D. S., Watson, G. O., Perryman, S., Nielsen, D. L. (2004): Ordination and significance testing of microbial community composition derived from terminal restriction fragment length polymorphisms: application of multivariate statistics. - Antonie van Leeuwenhoek 86: 339-347.

[22] Sergeant, M. J., Constantinidou, C., Cogan, T., Penn, C. W., Pallen, M. J. (2012): High-throughput sequencing of $16 \mathrm{~S}$ rRNA gene amplicons: effects of extraction procedure, primer length and annealing temperature. - PLoS ONE 7: e38094.

[23] Sul, W. J., Cole, J. R., Jesus, E. C., Wang, Q., Farris, R. J., Fish, J. A., Tiedje, J. M. (2011): Bacterial community comparisons by taxonomy-supervised analysis independent of sequence alignment and clustering. - Proceeding of the National Academy of Sciences of the United States of America 108: 14637-14642.

[24] Vasileiadis, S., Puglisi, E., Arena, M., Cappa, F., Cocconcelli, P. S., Trevisan, M. (2012): Soil bacterial diversity screening using single $16 \mathrm{~S}$ rRNA gene V regions coupled with multi-million read generating sequencing technologies. - PLoS ONE 7: e42671.

[25] Wang, Q., Garrity, G. M., Tiedje, J. M., Cole, J. D. (2007): Naïve Bayesian Classifier for Rapid Assignment of rRNA Sequences into the New Bacterial Taxonomy. - Applied Environmental Microbiology 73: 5261-5267

[26] Widder, S., Besemer, K., Singer, G. A., Geola, S., Bertuzzo, E., Quince, C., Sloan, W. T., Rinaldo, A., Battin, T. J. (2014): Fluvial network organization imprints on microbial co-occurrence networks. - Proceeding of the National Academy of Sciences of the United States of America 111: 12799-12804.

[27] Zhou, J. Z., Deng, Y., Zhang, P., Xu, K., Liang, Y., Van Nostrand, J. D., Yang, Y., He, Z., Wu, L., Stahl, D. A., Hazen, T. C., Tiedje, J. M., Arkin, A. P. (2014): Stochasticity, succession, and environmental perturbations in a fluidic ecosystem. - Proceeding of the National Academy of Sciences of the United States of America 111: E836-E845.

[28] Zhou, J., He, Z., Yang, Y., Deng, Y., Tringe, S. G., Alvarez-Cohen, L. (2015): High-throughput metagenomic technologies for complex microbial community analysis: open and closed formats. - mbio 6: e02288-14. 